\title{
Aristotelian Diagrams in the Debate on Future Contingents
}

\section{A Methodological Reflection on Hess's Open Future Square of Opposition}

\author{
Lorenz Demey
}

\section{Introduction}

In the recent debate on future contingents and the nature of the future, open theists such as Gregory Boyd (2010, 2011) and Molinists such as William Lane Craig $(1999,2011)$ have clarified their positions by appealing to certain logical notions, such as (the difference between) the Aristotelian relations of contradiction and contrariety. In an illuminating essay that was recently published in this journal, Hess (2017) argues in favor of Boyd's open theistic account, by constructing and defending an Aristotelian diagram which he calls the "open future square of opposition". Since this diagram "represents a logically coherent scenario" (Hess 2017, p. 14), Molinist arguments against open theism either fail, or end up begging the question.

My aim in this brief commentary paper is not to enter into this philosophical debate itself, but rather to highlight, at a more abstract methodological level, the important role that Aristotelian diagrams (such as Hess's square of opposition, but also others) can play in organizing and clarifying the debate. ${ }^{1}$ In particular, after providing a brief survey of the specific ways in which Boyd and Hess make use of Aristotelian relations and diagrams in the debate on the nature of the future, I will argue that the position of open theism is best represented by means of a hexagon of opposition (rather than a square of opposition, as Hess would have it). Next, I will show that on the classical theist account, this hexagon of opposition 'collapses' into a single pair of contradictory statements. This collapse from a hexagon into a pair of contradictories has several aspects, which can all be seen as different manifestations of a single underlying change (viz. the move from a tripartition to a bipartition of logical space).

\footnotetext{
${ }^{1}$ In Demey (2017) it is argued that Aristotelian diagrams can play a similar heuristic role in pedagogical contexts.
} 


\section{Boyd and Hess on Aristotelian Relations and Diagrams}

Boyd's $(2010,2011)$ defense of open theism is crucially based on his understanding of the Aristotelian relation holding between statements of the form " $x$ will obtain" and " $x$ will not obtain" (with $x$ standing for a state of affairs). ${ }^{2}$ He argues that these statements are not contradictory to each other, but rather contrary. In light of the well-known definitions of these relations, this means that although these two statements cannot be true together, they can be false together. Furthermore, the contradictory of " $x$ will obtain" is "not $[x$ will obtain]", which is equivalent to " $x$ might not obtain", and similarly, the contradictory of " $x$ will not obtain" is (equivalent to) " $x$ might obtain". The word "might" in the latter statements is not meant to capture the mere epistemic uncertainty of a finite knowing subject, but rather a genuine ontological possibility.

Based on these observations, Hess (2017) constructs an "open future square of opposition", which is shown here in Fig. 1a, and which serves as "a logical-semantic model of the openness of the future given Boyd's perspective" (2017, p. 1). The relations of contradiction and contrariety have already been dealt with in Boyd's remarks. Furthermore, since " $x$ will obtain" and " $x$ will not obtain" are contrary to each other, it follows that their contradictory statements, resp. " $x$ might not obtain" and " $x$ might obtain", are subcontrary to each other, i.e. they cannot be false together, but they can be true together. Similarly, it follows that there are subalternations from " $x$ will obtain" to " $x$ might obtain", and from " $x$ will not obtain" to " $x$ might not obtain". Hess explicitly makes use of these subalternations in his discussion with Craig, for example, when pointing out the "rather obvious truth" $(2017$, p. 5) that if "Claudius will go to the senate tomorrow" is true, then "Claudius might go to the senate tomorrow" is true as well.

\section{$<$ FIGURE 1 TO BE INSERTED HERE; SEE FINAL PAGE >}

Hess explicitly notes that this open future square of opposition has "the potential for three, not just two, logically distinct categories of future-oriented statements" (2017, p. 2, my emphases), viz. "will", "will not", and "might and might not". Although this threefold categorization is absolutely crucial in Hess's (and Boyd's) defense of open theism, I will argue in the next section

\footnotetext{
${ }^{2}$ Also see Rhoda et al. (2006), Hess (2017) and, for an older account, Hartshorne (1965).
} 
that he does not fully do justice to its importance, because of his choice to represent open theism by means of a square. Rather, the proper Aristotelian diagram to represent this threefold categorization is a hexagon of opposition.

\section{Open Theism: from Square to Hexagon}

Open theists are committed to the alethic openness of the future (Rhoda 2008, p. 229; Rhoda 2011, p. 74; Hess 2017, p. 5), i.e. the thesis that for some states of affairs $x$ and for some future times $t$, neither the statement " $x$ will obtain at $t$ " nor the statement " $x$ will not obtain at $t$ " is true. For open theists such as Boyd, Rhoda and Hess, who accept the principle of bivalence, this means precisely that the statements " $x$ will obtain at $t$ " and " $x$ will not obtain at $t$ " are both false. ${ }^{3}$ In light of the logical equivalences mentioned above, it follows that for some states of affairs $x$ and for some future times $t$, the statements " $x$ might obtain at $t$ " and " $x$ might not obtain at $t$ " are both true (note that this is an expression of the subcontrariety in Hess's square of opposition), and hence, their conjunction, " $x$ might obtain at $t$ and $x$ might not obtain at $t$ ", is true as well. ${ }^{4}$ The alethic openness of the future thus entails the logical consistency of this conjunction ("might and might not"). Hess asserts that "what the open future square of opposition is meant to show is that such a conception of ontology is in fact logically coherent [i.e. consistent] and therefore, representative of a possible way God might have decreed the future to be" (2017, p. 5). However, if the main point of Hess's square of opposition is to emphasize the consistency of the conjunction ("might and might not"), one can reasonably

\footnotetext{
${ }^{3} \mathrm{By}$ itself, the thesis of the alethic openness of the future is neutral with respect to the question whether the principle of bivalence applies to future contingents. Open theists such as Boyd, Rhoda and Hess retain bivalence; they hold that neither " $x$ will obtain at $t$ " nor " $x$ will not obtain at $t$ " is true, because both these statements are false. By contrast, open theists such as Tuggy (2007) reject bivalence for future contingents; they hold that neither " $x$ will obtain at $t$ " nor " $x$ will not obtain at $t$ " is true, because both these statements lack a truth value altogether (they are neither true nor false). See Rhoda (2008, p. 229; 2011, p. 75) for more discussion.

${ }^{4}$ Even when Hess (2017, p. 5) talks about the disjunction of these statements ("might or might not"), he emphasizes that this disjunction is inclusive in nature, and focuses on the case where it is true because both individual statements are true, thereby implicitly still getting at the conjunction after all (cf. "the conjoint truth of "might" and "might not"”, 2017, p. 6, my emphasis). Furthermore, elsewhere in the paper (e.g. on p. 6) Hess explicitly talks about the conjunction ("might and might not").
} 
wonder why this statement is not added to the square, next to the four statements that are already present in it.

Unlike the open theists mentioned above, Craig and other classical theists maintain that the future is alethically closed. This thesis is the logical negation of the alethic openness thesis, saying that for all states of affairs $x$ and for all future times $t$, one of the statements " $x$ will obtain at $t$ " and " $x$ will not obtain at $t$ " is true, and hence, their disjunction, " $x$ will obtain at $t$ or $x$ will not obtain at $t$ ", is true as well. ${ }^{5}$ Since this disjunction ("will or will not") is logically equivalent to the negation of the conjunction that we considered above ("might and might not"), it once again seems reasonable to add this statement to the square as well.

By adding both the conjunction of its subcontrary statements ("might and might not") and the disjunction of its contrary statements ("will or will not"), Hess's open future square of opposition has been expanded into a hexagon of opposition, which is shown in Fig. 2a. This Aristotelian diagram also contains some additional relations: (i) we have already seen that "will or will not" is logically equivalent to the negation of "might and might not", and hence both are contradictory to each other; (ii) we trivially have subalternations from the two disjuncts ("will", "will not") to their disjunction and from the conjunction ("might and might not") to both of its conjuncts; (iii) it is easy to show that "might and might not" is contrary to "will" as well as to "will not"; (iv) and similarly, that "will or will not" is subcontrary to "might" as well as to "might not". Since it explicitly contains the two statements corresponding to the alethic openness and alethic closedness of the future (and their Aristotelian relations to the four original statements), this hexagon of opposition arguably yields a much more helpful representation of (the debate between) open theism and classical theism.

\section{$<$ FIGURE 2 TO BE INSERTED HERE; SEE FINAL PAGE >}

One can show that there are no other (consistent) Boolean combinations of statements present in Hess's square of opposition. Logically speaking, this means that the hexagon of opposition

\footnotetext{
${ }^{5}$ As will be argued in the next section, the classical theist takes this disjunction ("will or will not") not only to be true, but even to be tautological in nature.

${ }^{6}$ Hess briefly hints at these two additional contrarieties, when noting that the "[con]junction [...] actually negates both "will" and "will not" propositions" (2017, p. 6); also see Footnote 4.
} 
is the Boolean closure of the square. As a consequence, it exhibits a highly systematic and 'symmetric' logical pattern, which is expressed by the following series of logical equivalences: ${ }^{7}$

(1) “will" $\Leftrightarrow$ "might" $\wedge$ "will or will not"

(2) "will not" $\Leftrightarrow$ "might not" $\wedge$ "will or will not"

(3) "might and might not" $\Leftrightarrow$ "might" $\wedge$ "might not"

(4) “might" $\Leftrightarrow$ "will” $\vee$ "might and might not"

(5) "might not" $\Leftrightarrow$ "will not" $\vee$ "might and might not"

(6) "will or will not" $\Leftrightarrow$ "will" $\vee$ "will not"

Furthermore, since this hexagon of opposition is Boolean closed, it represents an entire Boolean algebra of statements (except for the tautological and the contradictory statement, which are never shown in Aristotelian diagrams). This logical construction (from square to hexagon via Boolean closure) was first studied in the 1950s by the logicians Jacoby (1950), Sesmat (1951) and Blanché $(1953,1966)$, and the resulting hexagon is therefore nowadays often called the 'Jacoby-Sesmat-Blanché (JSB) hexagon' (Smessaert and Demey 2014). ${ }^{8}$

One can show that a (strong) JSB hexagon corresponds to a tripartition of logical space, with the three pairwise contrary statements constituting the 'cells' of the partition (Smessaert 2009). In the case of the JSB hexagon shown in Fig. 2a, this means that "will", "will not" and "might and might not" form a tripartition, i.e. for every state of affairs $x$, exactly one of these three statements applies. This is explicitly acknowledged by Hess, for example, when he writes that "according to the open future square [more correctly: hexagon] of opposition, there are three categories a putatively future event like Claudius' outing might fall into" $\left(2017\right.$, p. 6). ${ }^{9}$

\footnotetext{
${ }^{7}$ Also see Boyd (2010, p. 53) and Hess (2017, p. 2, Footnote 2). Note that equivalences (3) and (6) are trivial, because they merely involve the reformulation of the logical operations of $\wedge$ and $\vee$ into their natural language counterparts "and" and "or". Nevertheless, I have explicitly included equivalences (3) and (6) here, in order to emphasize their structural similarity to equivalences $(1-2)$ and $(4-5)$, respectively.

${ }^{8}$ It is interesting to note that the three discoverers of the JSB hexagon all worked in a distinctly religious (viz. Catholic) intellectual context. See Jaspers and Seuren (2016) for a detailed historical analysis of the broader cultural background to this observation.

${ }^{9}$ Also recall the quotation given in the final paragraph of the previous section.
} 
In logical geometry, i.e. the theoretical study of Aristotelian diagrams, the relevance of this underlying tripartition is made explicit by means of bitstring semantics. ${ }^{10}$ Informally, we work with bitstrings of length 3: the bitstring 100 corresponds to "will", the bitstring 001 corresponds to "will not", and the bitstring 010 corresponds to "might and might not". Based on logical equivalence (4) shown above, for example, it follows that "might" corresponds to $100 \mathrm{~V} 010$, i.e. to 110 . The complete bitstring semantics of the open theist's JSB hexagon is shown in Fig. 2b. We clearly see that this JSB hexagon contains all bitstrings of length 3 (except for 111 and 000, which correspond to the tautological and the contradictory statement; cf. supra), and thus represents the entire Boolean algebra $\{0,1\}^{3}=\{0,1\} \times\{0,1\} \times\{0,1\}$.

\section{Classical Theism: from Hexagon to Single Pair of Contradictories}

In the previous section I have argued that the subtleties of open theism are best represented by means of a JSB hexagon, because this diagram explicitly contains the notion "might and might not"; consequently, it is able to capture the open theist's tripartition of logical space by means of its full 'triangle of contraries'. However, one should ask what happens to this Aristotelian diagram if we move from the position of open theism (as defended by Boyd, for example) to that of classical theism (as defended by Craig, for example): is the JSB hexagon also the best representation of classical theism? After all, as Hess explicitly notes, "[w]hat Craig and other classical theists cannot affirm, however, is the full range of logical truths allowed by the open future square of opposition." (2017, p. 5). In particular, classical theism maintains that the conjunction " $x$ might obtain and $x$ might not obtain" does not constitute a genuine ontological possibility; in other words, this conjunction behaves logically as if it were the contradictory statement $\perp$. Similarly, the disjunction " $x$ will obtain or $x$ will not obtain" behaves logically as if it were the tautological statement $\mathrm{T}$.

These changes in logical behavior have a significant impact on the open theist's JSB hexagon. First of all, because "might and might not" and "will or will not" are no longer seen as contingent statements, they should no longer be shown in the Aristotelian diagram (cf. supra). This also has other consequences, which I will discuss based on the logical equivalences (1 -

\footnotetext{
${ }^{10}$ See Smessaert and Demey (2017) for a general overview of the applications of bitstring semantics, and Demey and Smessaert (2017) for its logical underpinnings.
} 
6) from the previous section. Systematically substituting $\perp$ and T for "might and might not" and “will or will not”, respectively, and doing some elementary logical reasoning, we obtain:

$$
\begin{aligned}
& \text { "will" } \Leftrightarrow \text { "might" } \wedge T \Leftrightarrow \text { "might" } \\
& \text { "will not" } \Leftrightarrow \text { "might not" } \wedge T \Leftrightarrow \text { "might not" } \\
& \perp \Leftrightarrow \text { "might" } \wedge \text { "might not" } \\
& \text { "might" } \Leftrightarrow \text { "will” } \vee \perp \Leftrightarrow \text { "will" } \\
& \text { "might not" } \Leftrightarrow \text { "will not" } \vee \perp \Leftrightarrow \text { "will not" } \\
& T \Leftrightarrow \text { "will" } \vee \text { "will not" }
\end{aligned}
$$

Equivalences $\left(1^{*}\right)$ and $\left(4^{*}\right)$ show that for the classical theist, "will" and "might" are logically equivalent to each other (whereas in the open theist's square/hexagon of opposition, there was a subalternation from "will" to "might"). Similarly, $\left(2^{*}\right)$ and $\left(5^{*}\right)$ show that for the classical theist, "will not" and "might not" are logically equivalent to each other (whereas in the open theist's square/hexagon, there was a subalternation from "will not" to "might not"). This is in line with Hess's remark that the classical theist takes "will not" and "might not" to be logically equivalent, and his defense of open theism based on "three separate lines of reasoning [...] in support of the idea that ["will" and "will not"] are, in fact, logically distinct propositions" (2017, p. 7). ${ }^{11}$ Next, equivalence $\left(3^{*}\right)$ expresses that for the classical theist, "might" and "might not" cannot be true together, and are thus contradictories (whereas in the open theist's square/hexagon, they were subcontraries). Finally, $\left(6^{*}\right)$ states that for the classical theist, "will" and "will not" cannot be false together, and are thus also contradictories (whereas in the open theist's square/hexagon, they were contraries). This is also noted by Hess, who writes that classical theism "requires that ["will" and "will not"] be construed as contradictory rather than contrary propositions" (2017, p. 7).

\footnotetext{
${ }^{11}$ In particular, Hess distinguishes between two different readings of the word "will" in the phrase "it will be the case that", viz. the posterior present reading and the simple future tense reading (the exact details of these two readings need not concern us here). He then goes on to argue, first, that "will" and "will not" under "the posterior present reading $[\ldots]$ can be seen to have different truth conditions, which means that they are not, strictly speaking, logically equivalent propositions" (2017, p. 8), and secondly, that they “- even on a simple future tense reading still are not logically equivalent statements" (2017, p. 10).
} 
By rejecting the open theist's assumption that the conjunction "might and might not" constitutes a genuine ontological possibility, the classical theist is thus required to radically re-think the configuration of logical relations holding between "will", "might" and related notions. These changes can be systematically summarized by stating that, whereas open theism corresponds to a JSB hexagon, classical theism can be represented by means of a single pair of contradictories. This (extremely simple) Aristotelian diagram is shown in Fig. 3a. Hess notes that "if [the classical theist] is right, then [...] the open future square does represent a false opposition" (2017, p. 9). In light of the diagrammatic analysis presented in this paper, Hess's remark can be reformulated more precisely as follows: if the classical theist is right, then the JSB hexagon collapses into a single pair of contradictory statements.

\section{< FIGURE 3 TO BE INSERTED HERE; SEE FINAL PAGE >}

These changes in logical behavior are all interrelated to each other. For example, Hess explicitly points out the interdependence of equivalences $\left(2^{*}\right),\left(5^{*}\right)$ and $\left(6^{*}\right)$ when writing that "if we read [the verb "will"] as simple future, ["will not" and "might not"] are logically equivalent [...] this means that, when taken in this (simple future) sense ["will" and "will not"] would be contradictories. Moreover, this appears to be Craig's interpretation of them as well." (2017, p. 9). The underlying explanation of all these changes is that by arguing that "might and might not" does not constitute a genuine ontological possibility, the classical theist effectively reduces the open theist's tripartition of logical space to a mere bipartition.

In order to illustrate this, I turn once more to the bitstring semantics that was introduced at the end of the previous section. We saw there that open theism can be represented by bitstrings of length 3, with the second bit position corresponding to "might and might not". Representing the position of classical theism thus requires us to delete this second bit position in the bitstrings. Systematically doing this to the bitstrings of the open theist's JSB hexagon in Fig. $2 b$ provides a unified explanation of all the logical changes that occur when we move from open theism to classical theism (the bit position to be deleted is systematically shown in boldface):

- "might and might not" is contingent for the open theist (010), but a contradiction for the classical theist (00) (and thus no longer shown in the diagram)

- "will or will not" is contingent for the open theist (101), but a tautology for the classical theist (11) (and thus no longer shown in the diagram) 
- there is a subalternation from "will" (100) to "might" (110) for the open theist, but they are logically equivalent (10 and 10) for the classical theist

- there is a subalternation from "will not" $(001)$ to "might not" $(011)$ for the open theist, but they are logically equivalent ( 01 and 01 ) for the classical theist

- there is a contrariety between "will" (100) and "will not" $(001)$ for the open theist, but they are contradictory (10 and 01$)$ for the classical theist

- there is a subcontrariety between "might" (110) and "might not" (011) for the open theist, but they are contradictory (10 and 01$)$ for the classical theist

The complete bitstring semantics of the classical theist's single pair of contradictory statements is shown in Fig. 3b. Just like the open theist's JSB hexagon in Fig. 2b, this Aristotelian diagram contains all bitstrings of length 2 (except for 11 and 00; cf. supra), and thus represents the entire Boolean algebra $\{0,1\}^{2}=\{0,1\} \times\{0,1\}$.

\section{Conclusion}

In this paper I have argued that Hess's (2017) open future square of opposition vacillates between two other Aristotelian diagrams. On the one hand, there is the open theist's JSB hexagon, i.e. the Boolean closure of the open future square itself, which represents the entire Boolean algebra $\{0,1\}^{3}$, and thus corresponds to a tripartition of logical space. On the other hand, there is the classical theist's single pair of contradictory statements, which represents the entire Boolean algebra $\{0,1\}^{2}$, and thus corresponds to a bipartition of logical space. ${ }^{12}$

In the theoretical investigation of Aristotelian diagrams, it is well-known that these diagrams are highly 'sensitive' to the underlying logical system, i.e. to one's logical assumptions (Demey

\footnotetext{
${ }^{12}$ In an unpublished manuscript entitled 'The Hexagon of Opposition: Thinking Outside the Aristotelian Box', Boyd, Belt and Rhoda anticipate several of the arguments presented in the current paper. In particular, they also develop a hexagon of opposition for representing the position of open theism, although they proceed in a different way than I do here. Furthermore, that paper does not provide a bitstring analysis of the hexagon, and does not discuss the 'collapse' from a JSB hexagon (for open theism) into a single pair of contradictory statements (for classical theism). Boyd, Belt and Rhoda's paper can be found online at http://reknew.org/2008/01/the-hexagonessay/ (accessed on 28 August 2017); I would like to thank an anonymous reviewer of this journal for bringing it to my attention.
} 
2015, Demey and Smessaert 2016, 2017, Pizzi 2016). The analysis in this paper shows that the same also holds for one's philosophical assumptions (in particular, regarding the question whether or not "might and might not" constitutes a genuine ontological possibility). It would be naïve to presume that Aristotelian diagrams can, by themselves, lead to a definitive solution of this philosophical debate. Nevertheless, I hope to have shown in this paper that these diagrams - and their corresponding bitstring analyses - can play an important methodological role, by systematically organizing and clarifying the debate.

\section{Acknowledgements}

I would like to thank Margaux Smets, Hans Smessaert and three anonymous reviewers for their useful feedback on an earlier version of this paper. The research presented in this paper was partially carried out during a research stay at the Faculty of Philosophy, University of Oxford, and is financially supported through a Postdoctoral Fellowship of the Research Foundation Flanders (FWO).

\section{References}

Blanché, R. (1953). Sur l'opposition des concepts. Theoria, 19, 89-130.

Blanché, R. (1966). Structures intellectuelles. Essai sur l'organisation systématique des concepts. Paris: Vrin.

Boyd, G. A. (2010). Two ancient (and modern) motivations for ascribing exhaustively definite foreknowledge to God: a historic overview and critical assessment. Religious Studies, 46, 4159.

Boyd, G. A. (2011). God limits his control. In D. W. Jowers (Ed.), Four views on divine providence (pp. 183-208). Grand Rapids, MI: Zondervan.

Craig, W. L. (1999). The only wise God: the compatibility of divine foreknowledge and human freedom. Eugene, OR: Wipf \& Stock Publishers. 
Craig, W. L. (2011). Response to Gregory A. Boyd. In D. W. Jowers (Ed.), Four views on divine providence (pp. 224-230). Grand Rapids, MI: Zondervan.

Demey, L. (2015). Interactively illustrating the context-sensitivity of Aristotelian diagrams. In H. Christiansen, I. Stojanovic, \& G. Papadopoulos (Eds.), Modeling and Using Context (pp. 331-345). Berlin: Springer.

Demey, L. (2017). Using syllogistics to teach metalogic. Metaphilosophy, 2017, 575 - 590.

Demey, L. \& Smessaert, H. (2016). Metalogical decorations of logical diagrams. Logica Universalis, 10, 233-292.

Demey, L. \& Smessaert, H. (2017). Combinatorial bitstring semantics for arbitrary logical fragments. Journal of Philosophical Logic, forthcoming.

Hartshorne, C. (1965). The meaning of 'is going to be'. Mind, 74, 46-58.

Hess, E. (2017). The open future square of opposition: a defense. Sophia, forthcoming.

Jacoby, P. (1950). A triangle of opposites for types of propositions in Aristotelian logic. New Scholasticism, 24, 32-56.

Jaspers, D. \& Seuren, P. A. M. (2016). The square of opposition in Catholic hands: a chapter in the history of 20th-century logic. Logique et Analyse, 59(233), 1-35.

Pizzi, C. (2016). Generalization and Composition of Modal Squares of Oppositions. Logica Universalis, 10, 313-325.

Rhoda, A. R. (2008) Generic open theism and some varieties thereof. Religious Studies, 44, 225 -234 . 
Rhoda, A. R. (2011). The fivefold openness of the future. In W. Hasker, T. J. Oord, \& D. Zimmerman (Eds.), God in an open universe: science, metaphysics, and open theism (pp. 6993). Eugene, OR: Pickwick.

Rhoda, A. R., Boyd, G. A., \& Belt, T. G. (2006). Open theism, omniscience, and the nature of the future. Faith and Philosophy, 23, 432-459.

Sesmat, A. (1951). Logique II. Les raisonnements. La syllogistique. Paris: Hermann.

Smessaert, H. (2009). On the 3D visualisation of logical relations. Logica Universalis, 3, 303332.

Smessaert, H. \& Demey, L. (2014). Logical geometries and information in the square of oppositions. Journal of Logic, Language and Information, 23, 527-565.

Smessaert, H. \& Demey, L. (2017). The unreasonable effectiveness of bitstrings in logical geometry. In J.-Y. Béziau \& G. Basti (Eds.), The square of opposition: a cornerstone of thought (pp. 197-214). Basel: Springer.

Tuggy, D. (2007). Three roads to open theism. Faith and Philosophy, 24, 28 - 51. 
(a)

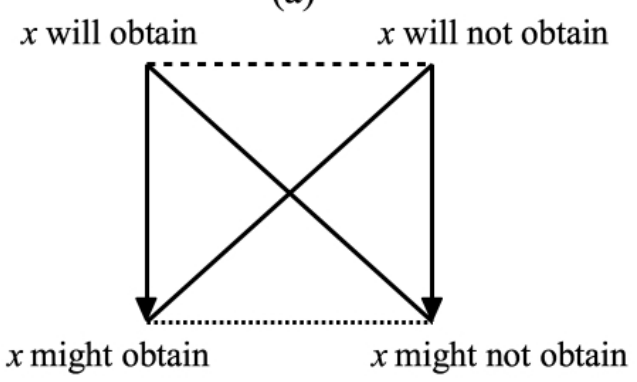

(b)

contradiction

contrariety

subcontrariety

subalternation

Fig. 1: (a) Hess's open future square of opposition, (b) code for visualizing the Aristotelian relations.

(a)

$x$ will obtain or

$x$ will not obtain

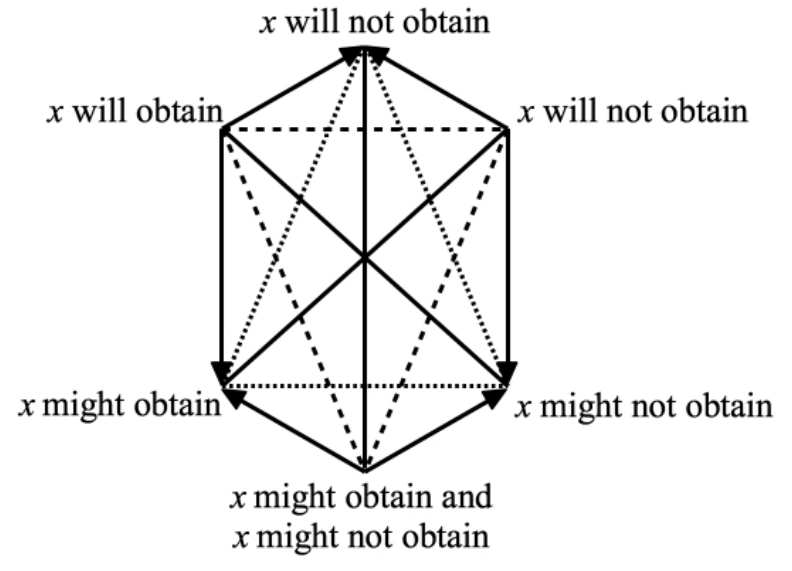

(b)

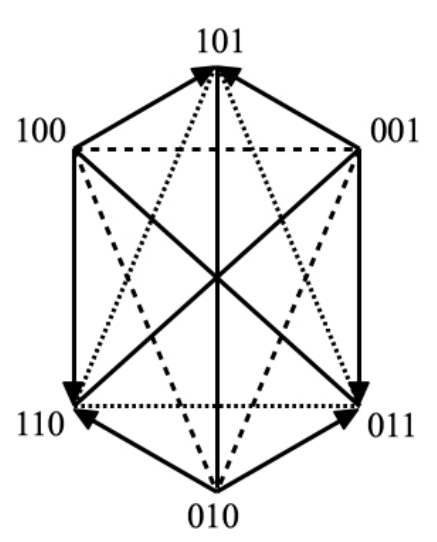

Fig. 2: (a) the open theist's JSB hexagon, (b) its bitstring semantics.

(a)

$x$ will obtain $x$ might obtain $x$ will not obtain

$x$ might not obtain (b)

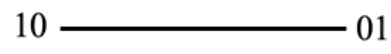

Fig. 3: (a) the classical theist's single pair of contradictories, (b) its bitstring semantics. 